\title{
The structure of visual pattern associates and pattern goodness'
}

STEPHEN HANDEL AND W. R. GARNER

JOHNS HOPKINS UNIVERSITY
Two tasks were used with a total set of 126 dot patterns. In one task $S$ s rated the goodness of each pattern. In another task they produced a dot pattern as an associate to each of the patterns used as a stimulus. The distributions of the associates suggest that the total set of patterns is both partitioned and nested. Groups defined by rotation and reflection are partitioned, thus kept intact. These groups in turn form a series of nested subsets. Both partitioning and nesting produce subsets of different size. The size of these subsets is related to pattern goodness, with good patterns coming from small subsets

Garner (1962) hypothesized that when an observer perceives a particular pattern he infers a subset of patterns which are equivalent to it and that the size of this inferred subset is inversely related to pattern goodness, i.e., good patterns come from small inferred subsets. Garner \& Clement (1963) used 90 dot patterns in a direct test of this hypothesis. They obtained ratings of the goodness of each of these patterns, and also required Ss to divide the total set of patterns into mutually exclusive groups according to a similarity criterion. The ratings of the patterns were highly correlated with the size of the groups in which the patterns had been placed, a result giving strong support to the hypothesis. The instructions given Ss in that experiment were to form a simple partition of the 90 patterns. Since similar patterns were to be kept together, we can assume that patterns within a group are more similar (more closely related) to each other than they are to patterns in other groups. A simple partition allows only one relationship: All patterns must be interpreted as either similar or dissimilar. Furthermore, the similarities within a group must be considered completely symmetrical. Pattern associates.

A simple task requiring $S$ s to produce patterns as associates to other patterns can reveal more subtle and complex ways in which subsets of varying size can be formed. We can define a complete set of patterns which serve as stimuli and we can restrict the associates to this same complete set. We have a closed, and thus interpretable, system of stimuli and associates. The data can be displayed in a square matrix in which the columns represent the patterns used as stimuli and the rows represent the same patterns used as associates.

Each stimulus pattern will have a set of patterns which are commonly used as associates. We assume that the associates to a pattern define the psychologically equivalent or inferred set of that particular pattern. In other words, we assume that all associates come from the inferred subset of that pattern.

\section{Profotypical Outcomes}

While the number of possible outcomes of such an experiment is essentially limitless, it is worth considering the implications of a few prototypical outcomes. Uniform distribution.

If all patterns are used equally often as associates to all stimuli, we have no evidence of the existence of inferred subsets, since this outcome implies that all patterns are equally available as associates to each stimulus. It is also possible that some patterns will be used as associates more often than others, but that this unequal distribution is the same for all stimuli. Such an outcome would suggest the operation of a simple response bias, but would still not provide evidence of the existence of inferred subsets.

Simple partitioning.

A second prototypical outcome results from simple and complete partitioning, i.e., there are subsets which are mutually exclusive and which may differ in size. (This is the only outcome which the grouping task used by Garner and Clement allowed.) With simple partitioning, the data matrix can be arranged so that groups of successively arranged patterns use each other as associates, never using patterns from different groups as associates. The crucial part of this prototypical outcome is the mutual exclusion: Subsets must relate within themselves, but not interact with other subsets. The particular configuration of associates within the subset is not important, and certainly uniform or symmetric distributions of associates within subsets a re not required. To illustrate, suppose pattern $B$ is used as the associate to $A, C$ is used as the associate to $B, D$ is used as the associate to $C$ and $A$ in turn is used as the associate to $D$. If none of these patterns is used as an associate to any other pattern, we have a partitioned subset since all other patterns are excluded, but we do not have a uniform or symmetric distribution.

Simple nesting.

A third prototypical outcomeoccurs when the patterns form subsets which are nested or included within one another. An example of a series of nested sets is: the set of all humans, the set of all male humans, the set of all male humans with red hair, the set of all male humans with red hair and blue eyes. The nesting concept is potentially more valuable in understanding the relation of set size to pattern goodness because it, unlike the partitioning concept, guarantees that the subsets will differ in size. All partitioned subsets can be equal, but nested subsets must be progressively smaller. We assume, as with simple partitioning, that the 
associate comes from the subset which is inferred from the stimulus pattern. With nested subsets, however, a pattern can be in many different subsets. We shall assume, then, that the associate must come from the smallest subset inferred for any pattern. An alternative way of stating this assumption is to say that $S$ always infers the smallest subset appropriate to any stimulus pattern.

It is possible for the nesting concept to be correct even though the assumption that associates come from the smallest subset is incorrect. In such a case the data from an associates experiment give us no information. If, however, both the nesting concept and the assumption concerning associates a re correct, there are two clear implications concerning the matrix of associates.

First, it means that the associates will be asymmetrical. To illustrate, suppose pattern $\mathrm{A}$ is in the total set but in no smaller subset. Since its smallest subset is the total set, it can have any pattern as an associate to it. Now suppose pattern B is in the smallest subset. It will have associates only from this smallest subset, and that subset does not include pattern $A$. Thus pattern $B$ can be an associate to pattern $A$, but $A$ cannot be an associate to $\mathbf{B}$.

Complete asymmetry means that the patterns can be arranged in the matrix so that all associates fall above a diagonal line, giving a triangular matrix. The ordering of patterns which produces the triangular matrix would be the actual nesting order of the patterns. This nesting order will in turn determine the order of the sizes of the inferred subsets, since the most included patterns are in the smallest subsets.

Second, the nesting concept means that all patterns will not be used equally often as associates. Those patterns which are in many different subsets can be used as associates to many different stimulus patterns, and will therefore be used very frequently. Conversely, those patterns which are in few subsets will be used as associates to few stimulus patterns, and will therefore be used infrequently. This unequal frequency of use is similar to the result expected with a simple response bias, except that the nesting concept does not allow the same distribution of associates to each stimulus. Purpose of experiment.

The specific purpose of this experiment is to obtain pattern associates to a large set of patterns in order to investigate the structure of the inferred subsets. In keeping with the original hypothesis, the sizes of these subsets are compared to rated goodness of the patterns in the expectation that good patterns will come from small inferred subsets.

\section{Method}

\section{The Stimuli}

The stimulus patterns consisted of all 126 possible arrangements of five dots in the cells of a $3-b y-3$ matrix. Each cell of the matrix was 0.5 in by 0.5 in.
The dot was 0.25 in in diameter, and was placed in the center of the cell. Both the dot and the matrix were printed in black on a light blue card.

R-R groups.

Representative patterns are shown in Fig. 1. Each pattern shown is from a group of patterns. Each pattern in the figure can produce the remaining patterns in its group by rotation and/or reflection. The number in the pattern code indicates the number of equivalent patterns, and the letter indicates the particular pattern. To illustrate, pattern $1 \mathrm{a}$ is unique because any 90-degree rotation or reflection simply produces the same pattern. Pattern 4a, however, consists of four equivalent patterns because there are four patterns which can produce each other by reflection and rotation. Likewise, all patterns whose code number is 8 exist in groups of eight equivalent patterns. Such groups of stimuli will be called R-R groups.

\section{Tasks and Subjects}

Goodness ratings.

Twenty Ss randomly selected from the introductory psychology course at Johns Hopkins were required to make ratings of all 126 patterns on a seven-point scale, with " 1 " being very good and " 7 "' very poor. 2 Each $S$ had a different random order of the stimuli, and the judgments were written on the side of the stimulus card. Ss were allowed to examine a few patterns to insure a frame of reference, but after this orientation they worked continuously without being allowed to look back. The task took about $30 \mathrm{~min}$. and Ss were self-paced. Pattern associates.

A different $126 \mathrm{Ss}$ from the same source were used in the associates task. Each $\mathrm{S}$ produced an associate to each of the 126 patterns used as a stimulus. A latin square counterbalancing order was used, and the square was also arranged so that each pair sequence occurred equally often. (This counterbalancing arrangement was used on the assumption that there might be some order effects. Comparison of the first and last ten trials showed such effects to be trivial, however, and no further reporting of sequence effects will be made.)

For this task, each pattern was placed on the left side of a 3 in by 8 in card, and there was a blank matrix of the same size on the right of the card, in which $\mathrm{S}$ was required to "draw a pattern of five dots, which is suggested by, but different from, the pattern in the left-hand matrix." Ss were allowed to make any sort of mark in the cells of the matrix, rather than being required to make a neat circle. The 126 cards were arranged in a deck, and $\mathrm{S}$ worked down from the top of the deck, turning each card over as he finished. Ss were self-paced and nearly all of them finished in 20 $\min$.

A critical aspect of the instructions was that only patterns with five dots could be used as associates. This instruction insured that the set of stimulus patterns was the same as the set of associates, since all possible pat- 


\begin{tabular}{|c|c|c|c|c|}
\hline \multicolumn{2}{|c|}{$\begin{array}{c}\text { CODE } \\
\text { AND }\end{array}$} & $\begin{array}{c}\text { MEAN } \\
\text { GOODNESS }\end{array}$ & $\begin{array}{c}\text { MEAN } \\
\text { ASSOCIATE }\end{array}$ & NESTING \\
\hline \multicolumn{2}{|c|}{ PATTERN } & RATING & FREQUENCY & ORDER \\
\hline 10 & 6010 & 1.09 & 3.68 & 1 \\
\hline $1 \mathrm{~b}$ & & 1.14 & 3.22 & 2 \\
\hline 40 & :日0 & 1.80 & 1.86 & 4 \\
\hline $4 t$ & 足1 & 1.99 & 1.73 & 3 \\
\hline $4 c$ & $\because 0$ & 2.06 & 0.86 & 14 \\
\hline $4 d$ & 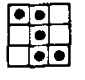 & 2.30 & 1.58 & 5 \\
\hline $4 \mathrm{e}$ & & 2.43 & 1.48 & 8 \\
\hline $4 f$ & मेन्व & 2.63 & 1.19 & 10 \\
\hline $4 \mathrm{~g}$ & & 2.82 & 1.21 & 11 \\
\hline $4 \mathrm{~h}$ & & 2.83 & 1.46 & 7 \\
\hline $4 i$ & & 2.94 & 0.83 & 15 \\
\hline $4 j$ & alo & 3.57 & 0.78 & 12 \\
\hline $4 k$ & 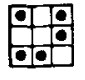 & 4.51 & 0.47 & 23 \\
\hline 80 & :-10 & 3.52 & 1.15 & 9 \\
\hline $8 \mathrm{~b}$ & $\begin{array}{lll}\div 00 \\
\div 0\end{array}$ & 3.55 & 1.68 & 6 \\
\hline $8 c$ & & 3.93 & 0.63 & 19 \\
\hline $8 d$ & & 4.04 & 1.09 & 13 \\
\hline $8 e$ & & 4.90 & 0.53 & 21 \\
\hline $8 f$ & & 4.92 & 0.76 & 16 \\
\hline 89 & & 5.16 & 0.55 & 20 \\
\hline $8 \mathrm{~h}$ & प0 & 5.41 & 0.50 & 22 \\
\hline $8 i$ & 0 & 5.55 & 0.64 & 17 \\
\hline $8 j$ & ato & 5.63 & 0.57 & 18 \\
\hline
\end{tabular}

Fig. 1. Three measures related to the various dot patterns. (The first number in the pattern code indicates the number of equivalent pattems by rotation and reflection, and the letter indicates, in order, the rated goodness of the pattems within each size of R-R group. See text for explanation of other measures. terns were in fact used. A total of 15,876 pattern associates was obtained, and only 87 of them were not useable because they did not come from the defined set of possible patterns.

The instructions also stated that the associate was to be different from the stimulus pattern. A small number of the associates were the same as the stimulus, but these associates were kept in the analysis.

\section{Results: Goodness Ratings}

The mean goodness rating for each of the $R-R$ groups of patterns is given in Fig. 1. An analysis of variance of these ratings was carried out, and $98 \%$ of the variance is due to differences between the $23 \mathrm{R}-\mathrm{R}$ groups. Thus patterns which are the same when rotated or reflected are given the same goodness rating. Furthermore, the rated goodness is highly related to the size of the $R-R$ group. The patterns are almost perfectly ordered by size of group, with only patterns $4 j$ and $4 k$ overlapping the rated goodness of the groups with eight equivalents.

These patterns are essentially the same as those used by Garner and Clement, except that patterns $4 e, 4 f$, $4 \mathrm{i}, 8 \mathrm{a}, 8 \mathrm{~b}$, and $8 \mathrm{c}$ had been omitted in the earlier experiment, since the missing row or column in these patterns makes their orientation difficult to maintain when the actual grid lines of the matrix are not shown. They were included in the present experiment so that the set of associates would be the same as the set of stimuli.

The inclusion of these additional patterns had very little effect on the rated goodness. Garner and Clement had found the same percentage of variance accounted for by the R-R groups. Furthermore, the rank correlation coefficient for the ratings of the $17 \mathrm{R}-\mathrm{R}$ groups which were used in both experiments is .98 . Thus these additional patterns and the grid lines do not change the nature of pattern goodness as found in the earlier experiment.

\section{Results: Pattern Associates}

The basic data from the associates task formed a matrix with $126-$ by-126 cells. It was clear from this matrix that both partitioning and nesting processes were operative in determining the nature of the associates. Furthermore, the partitioning occurred primarily with respect to the $R-R$ groups of patterns. Therefore, the total matrix has been reduced to a matrix of the $23 \mathrm{R}-\mathrm{R}$ groups, and this matrix, in simplified form, is shown in Table 1.

In this matrix, each number is an average frequency of associate calculated from the original complete matrix. Since the sizes of the R-R groups are either 1,4 , or 8 , the number of cells in the complete matrix represented by a single cell in the reduced matrix can be $1,4,8,16,32$, or 64 , depending on the size of the stimulus pattern group and the size of the associate pattern group. The number in Table 1 is the average for all cells represented. Each number is the lower bound 
Table I. Schematized Matrix Showing Average Frequency with Which Various Pattems are Used as Associates to the Patterns Presented as Stimuli

\begin{tabular}{|c|c|c|c|c|c|c|c|c|c|c|c|c|c|c|c|c|c|c|c|c|c|c|c|}
\hline & & & & & & & & & & & imuls & ${ }_{s} \mathrm{~Pa}$ & tern & & & & & & & & & & \\
\hline & la & $\mathrm{Ib}$ & $4 \mathrm{~b}$ & $4 a$ & $4 d$ & $8 b$ & $4 \mathrm{~h}$ & $4 e$ & $8 a$ & $4 f$ & $4 g$ & $4 j$ & $8 \mathrm{~d}$ & $4 c$ & $4 \mathrm{i}$ & $8 \mathrm{f}$ & $8 i$ & $8 i$ & $8 c$ & $8 g$ & $8 e$ & $8 \mathrm{~h}$ & $4 k$ \\
\hline la & & 58 & 2 & 12 & 3 & 1 & 1 & 1 & 3 & 1 & 2 & 8 & & 2 & 6 & 13 & 1 & 2 & 2 & & 1 & & \\
\hline $\mathrm{ib}$ & 57 & & 3 & 2 & 4 & $i$ & 4 & 2 & & 1 & 2 & 8 & 4 & 4 & 9 & 1 & 10 & 1 & 1 & 1 & & & 1 \\
\hline $4 b$ & & & 23 & 1 & & 1 & 1 & & 1 & & 1 & & & & & & & & & 2 & 2 & 1 & 3 \\
\hline 40 & 4 & 1 & 1 & 20 & 1 & 1 & & 2 & & 2 & & & 3 & 2 & 2 & 1 & 1 & 1 & 2 & & 1 & & \\
\hline $4 d$ & 1 & 1 & & & 22 & & & & & 1 & 2 & & & & & 2 & 1 & & $\mathrm{I}$ & 1 & & 2 & \\
\hline $8 \mathrm{~b}$ & & & & & & 8 & 2 & 1 & 4 & & 1 & 1 & 1 & & & & & 1 & 2 & 1 & 1 & & \\
\hline 4h & & 1 & 1 & & & 1 & 19 & & 1 & & 2 & 1 & & & & & 1 & 1 & & & & 1 & 1 \\
\hline $4 e$ & & & & 1 & & 1 & & 18 & & 4 & & & & 2 & 3 & & & & & 2 & & 2 & \\
\hline $8 e$ & & & & & & $i$ & & & 6 & & & 1 & & & & 1 & & 1 & 1 & & & & \\
\hline $4 f$ & & 1 & & & 2 & & & 3 & & 15 & & & & 3 & 1 & & 1 & & 1 & & & & \\
\hline $4 \mathrm{~g}$ & & & 1 & & 1 & & & & & & 13 & & & & & 1 & & 3 & & & & 1 & 1 \\
\hline $4 j$ & & 1 & & & & & & & & & & 15 & & 1 & & & & & & & & 1 & \\
\hline $8 d$ & & & & & & & & & & & & & 8 & & & & 1 & & & 1 & 1 & & \\
\hline $4 c$ & & & & & & & & & & 1 & & & & 17 & & & & & & & & & 1 \\
\hline $4 i$ & & 2 & & & & & & 2 & & 1 & & & & & 16 & & & & & & & & \\
\hline $8 f$ & & & & & & & & & & & & & & & & 6 & & & & & & & \\
\hline $8 i$ & & & & & & & & & & & & & & & & & 6 & & & & & & \\
\hline $8 \mathrm{i}$ & & & & & & & & & & & & & & & & & & 5 & & & & & \\
\hline $\begin{array}{l}8 c \\
B_{g}\end{array}$ & & & & & & & & & & & & & & & & & & & 7 & 6 & & & 1 \\
\hline $8 \mathbf{e}$ & & & & & & & & & & & & & & & & & & & & & 6 & & \\
\hline $8 \mathrm{~h}$ & & & & & & & & & & & & & & & & & & & & & & 6 & \\
\hline $4 k$ & & & & & & & & & & & & & & & & & & & & & & & 12 \\
\hline R.R\% & & & 56 & 48 & 52 & 48 & 47 & 45 & 35 & 36 & 32 & 38 & 50 & 41 & 39 & 34 & 35 & 30 & 41 & 37 & 35 & 36 & 31 \\
\hline
\end{tabular}

Note. Each cell entry is the average frequency for all cells from the 126-by-126 matrix represented in each cell of this matrix. The number is the lower bound for unit class intervals. The numbers on the bottom row are the average percentages of assaciates which are equivalent to other patterns by rotation and reflection.

of the interval represented, so that a "1" means any frequency from 1.0 up to 2.0 , etc. A value of 1.0 is the expected value if all patterns are used equally often as associates regardless of the stimulus, i.e., if there is a uniform distribution.

\section{Partitioning Processes}

\section{R-R groups.}

The goodness ratings in both this experiment and the Garner-Clement experiment showed that all patterns in the same $\mathbf{R}-\mathbf{R}$ group have the same rated goodness. Analysis of the partitions formed in the Garner-Clement experiment also showed a strong tendency for Ss to keep R-R groups intact. Thus, we can expect the $R-R$ groups to operate as partitioned subsets with respect to the pattern associates.

The frequencies on the diagonal of the matrix in Table 1 show how often, on the average, the associate to a pattern was from its own R-R group. (The number of cells from the complete matrix represented by each diagonal cell in Table 1 differs from the number in non-diagonal cells because $S$ was not permitted to use the stimulus pattern as the associate. Thus there are no entries on the diagonal for patterns $1 \mathrm{a}$ and $1 \mathrm{~b}$. To illustrate, each pattern in subset 4 a has three associates which remain in the $R-R$ subset, so that the diagonal frequency in this case is an average from 12 cells in the complete matrix.)

These diagonal frequencies are usually very large, and $39.1 \%$ of the total number of associates were to another pattern within the same $\mathbf{R}-\mathbf{R}$ group. This value can be compared to the value of $4 \%$ expected from a uniform distribution. In other words, almost 10 times as many associates remain within the same $R-R$ group as we would expect on a pure chance basis.

In some cases, the associates within the $\mathrm{R}-\mathrm{R}$ group indicate complete symmetry, and in the other cases there are various partial chaining effects, even occasionally complete circling. These effects operate particularly when the patterns have strong vertical or horizontal properties.

Relation to goodness.

We have already noted that the size of the (partitioned) R-R group is related to goodness of the patterns. A still further relation to goodness can be seen from the individual percentages of use of the different patterns in the same R-R group as associates, shown at the bottom of Table 1. These percentages have a rank correlation of .73 , with the rated pattern goodness (in Fig. 1), indicating that the better the pattern the more likely are Ss to use another pattern from the same $\mathbf{R}-\mathbf{R}$ group as an associate.

This relation is not only intuitively reasonable in that good patterns would be expected to be used more frequently, but it is essential if a nesting process operates. The nesting process assumes that associates are in the smallest inferred set. If the best patterns are in smaller, more nested, subsets, there are fewer possible R-R groups which can be used as associates. Thus, more responses should be made to other patterns in the 
same R-R group.

\section{Nesting Processes}

As indicated above, if subsets are nested within the total set, there are two important consequences relating to the matrix of associates: The associates should be asymmetrical, and the various patterns should be used with unequal frequency as a consequence of the asymmetry.

Asymmetry of associates.

The original matrix of stimulus-associates has no necessary ordering of the rows and columns. If there is substantial asymmetry in the pattern of associates, then it should be possible to arrange the rows and columns into the same order so as to have a majority of the associates above the diagonal.

The order of the patterns in Table 1 (also shown in Fig. 1) is the order which maximizes the number of associates above the diagonal. A scalogram technique for ordering items in a test (Green, 1954) was used to obtain an initial order of patterns, and then additional adjustments were made to obtain a maximum number of associates above the diagonal. Table 1 shows the extent to which this order does produce a high proportion of associates above the diagonal. This matrix shows all cell entries which exceed the expected value of 1.0 . There are 105 entries above the diagonal compared to 23 below the diagonal, but this ratio becomes even greater when comparison is made with cells of greater frequency. For example, there are 22 cells above the diagonal with average frequencies 3 or greater, compared to just 3 below.

In terms of total frequencies, 7,093 associates occurred above the diagonal compared to 2,522 below the diagonal. These total frequencies can also be arranged according to the size of the R-R groups, and they are shown in Table 2. The diagonal frequencies in this case include the associates to the same R-R groups as well as to other groups of the same size. Here the strong tendency for the associate to come from a smaller group can be seen again. The majority of patterns from unique groups (1a and $1 \mathrm{~b})$ produce the other unique pattern as associate (plus nine errors in which the same pattern is produced). The patterns from groups of 4 produce a large number of associates from other groups of 4 , but relatively few from groups of 8 , even though there are 80 such patterns compared to only 44 patterns from groups of 4 . A disproportionately large number of associates from the smaller groups also occurs with patterns from groups of 8 .

Table 2 can be considered to provide the basis for the transitional probabilities of a Markov pròcess in which each associate becomes the stimulus for the next associate in an infinite chain of associaies. This process is analogous to a successive reproduction experiment. With these transitional probabilities, the chain would ultimately produce stable first-order probabilities for the different patterns, and the asymptotic first order probability that one of the two unique pat-
Table 2. Total number of associates given to various stimulus patterns according to the size of the $\mathbf{R}-\mathbf{R}$ groups

\begin{tabular}{lrrrrr} 
& \multicolumn{5}{c}{ Size of Stimulus Group } \\
Size of & 1 & 1 & 4 & 8 & Totol \\
Associate & 4 & 76 & 338 & 407 & 869 \\
Group & 8 & 46 & 1,931 & 2,762 & 6,769 \\
Total & & 246 & 5,520 & 10,023 & 15,789
\end{tabular}

terns would occur is .10 . Since only two unique patterns exist in the total set of 126 , the strength of the asymmetry of associates can be seen very clearly.

Frequency of associates.

The mean frequency with which each pattern in each R-R group was used by each $\mathrm{S}$, regardless of which pattern was used as stimulus, is shown in Fig. 1. The expected value for this measure, assuming uniform distribution, is 1.0, and these values range both well above and below that value. So there are substantial differences in frequency of use of the various patterns as associates, as required by the nesting concept. Furthermore, the rank correlation between the mean frequency and the nesting order is .99 , a value necessarily high.

A simple response bias (even if due to preference for good patterns) could produce the unequal frequency of use with no implication of a nesting process. However, Table 1 makes it very clear that these results are not due to simple response bias. The crucial point is that the pattern of associates is not the same for all stimulus patterns, and differs in a way compatible with a nesting concept. The nesting concept would predict that the patterns at the outside of the nesting order show the least use of the unique R-R patterns, since there are many patterns available as associates. Furthermore, there are some highly specific patterns of associates, clearly due to the particular configuration of the patterns involved and not due to an overall preference for some patterns. For example, pattern $8 i$ frequently produces pattern $1 \mathrm{~b}$ as an associate and rarely $1 \mathrm{a}$, while the converse is true for pattern $8 \mathrm{f}$, and both of these relations are obvious from the nature of the patterns. Therefore, while we cannot argue that no simple response bias exists, we can argue that there are factors in addition to any simple bias.

Relation to goodness.

Presumably those patterns nested furthest inside, such as patterns $1 a, 1 b$, and $4 b$, are in the smallest subsets, and those nested outside, such as $8 \mathrm{e}, 8 \mathrm{~h}$, and $4 \mathrm{k}$, are in the largest subsets. If the original hypothesis that good patterns come from small subsets is true, then the nesting order should be correlated with rated goodness. The nesting order has a rank correlation with goodness ratings of $\mathbf{8 5}$, and the mean associate frequency has a rank correlation with goodness of .87 , so our requirement of correlation between size of nested set and goodness is satisfied. The major discrepancies between these measures appear to be related to response bias. Patterns $4 \mathrm{c}$ and $4 \mathrm{k}$ are not used as a major associate to any other pattern, and 
thus are nested in large subsets, although both have much better ratings when presented as stimuli.

\section{Conclusions}

These experimental results can be summarized as follows: Both partitioning and nesting processes function in determining the size of inferred subsets of patterns. Groups produced by the objective criteria of rotation and reflection constitute partitions of different sizes, and these partitions are reflected in the Ss'associates. These R-R groups in turn appear to form a series of nested subsets, with their necessary variation in size. Regardless of whether the variation in size is produced by partitioning or the independent process of nesting, the subset size is inversely correlated with pattern goodness.

The asymmetry relation is a fundamental property of nested sets, in that the inclusion relation is asymmetrical. In this experiment, asymmetry of associates was found. Other types of asymmetry can exist. For example, Bresson \& Vurpillot (1960) found that errors made in reproducing tachistoscopically presented patterns were asymmetrical. And the errors were toward more regular patterns. Also, many studies have found that remembered patterns are changed in the direction of better patterns. These studies show an asymmetrical relation since the patterns change unidirectionally. In all of these cases the nesting idea is useful as an explanatory concept, and is an explanation which requires the additional idea of different sizes of subsets. The smaller the subset, the greater is the structure, or redundancy, relative to the total set. Therefore, those psychological processes which show asymmetry or unidirectionality, and which can thus be interpreted in terms of a nesting concept, are showing change in the direction of increased structure and redundancy.

\section{References}

Bresson, F., \& Vurpillot, E. Contribution à une psychophysique des formes. Psych. francaise, 1960, 5, 29-45.

Garner, W. R. Uncertainty and structure as psychological concepts. New York: Wiley, 1962.

Garner, W. R. \& Clement, D. E. Goodness of pattern and pattern uncertainty. J. verbal Learn. verbal Behav., 1963, 2, 446-452.

Green, B. F. A method of scalogram analysis using summary statistics. Psychometrika, 1956, 21, 79-89.

\section{Notes}

1. This research was supported in part by Grant No, 1 RO1 MH11062 from the National Institute of Mental Health to Johns Hopkins University.

2. We are grateful to Richard Degerman for collection of the goodness rating data. 